\title{
International Chemical Identifier
}

National Cancer Institute

\section{Source}

National Cancer Institute. International Chemical Identifier. NCI Thesaurus. Code C54683.

A textual identifier for chemical substances designed to provide a standard and humanreadable way to encode molecular information that also facilitates searches in printed and electronic data sources. 